\title{
FUNDAMENTOS DE LA EDUCACIÓN SUPERIOR DEL EJÉRCITO DE CHILE
}

Departamento I "Académico Docente"

Jefatura de Docencia

Comando de Institutos Militares 


\section{RESUMEN}

El ejército, frente al desafío de estructurar un sistema educacional acorde con las exigencias del siglo XXI, difundió en 1997 la publicación: "Sistema Educativo Institucional: Filosofía, Políticas y Alcances", que contiene los fundamentos de la educación militar de calidad y las estrategias para su aplicación, en el marco de la reforma educacional. Posteriormente, el año 2000, se publicó un segundo libro sobre el "Sistema Educativo Institucional: Gestión Docente", continuación y complemento del texto anterior.

El propósito de este artículo es dar expresión a los fundamentos de la educación militar. Para ello se presentará una visión de carácter global, basada en ambos textos, del modelo institucional en el marco de la modernización.

\section{ABSTRACT}

Facing the challenge to structure an educational system in harmony with the demands of the XXIst century, the army published, in 1997, the "Institutional Educational System: Philosophy, Politics and Ranges"; it contains the basis of the military education and the strategies for its management, according to the educational reform. A second book was published in the year 2000: "Institutional Educational System: Teaching Management", a complement to the previous one.

The purpose of this study is to express the army's fundamental educational basis. A global vision of the institutional model within the frame of modernisation will be given, based on both texts. 


\section{FUNDAMENTOS DE LA EDUCACIÓN SUPERIOR DEL EJÉRCITO DE CHILE}

\section{MODELO INSTITUCIONAL.}

\section{BASE DEL SISTEMA EDUCACIONAL ${ }^{1}$}

Un aspecto esencial que hay que considerar, básico en cualquier proyecto relacionado con la carrera militar, es el modelo de profesionalidad que sigue la institución militar. Ello implica profundizar los aspectos fundamentales de su doctrina y, en general, todo aquello que se vincula con el sentido y la forma en que una institución en particular aborda su quehacer.

A través del tiempo los modelos de profesionalidad han variado. En ello ha influido la realidad particular de cada país, su desarrollo tecnológico, las amenazas que debe enfrentar y, por cierto, la historia, tradiciones e identidad de la propia institución. Así, hay ejércitos que adscriben a un modelo ocupacional en el que lo esencial son los valores plasmados en la eficacia: el interés propio se antepone al interés de la institución y el nivel de destreza, conocimiento y aptitudes, así como la retribución monetaria, son de máxima importancia. Otros, en cambio, enfatizan un modelo en el que los valores individuales se subordinan a un bien común que se asume en el largo plazo: la permanencia en la institución es valorada, se refuerza la identificación con el ejército y la patria, y se otorga menos importancia al componente retributivo.

1 Comando de Institutos Militares, Sistema Educativo Institucional: Filosofía, Políticas y Alcances, Santiago, Chile, 1997, pp. 31-32. 
En el caso del Ejército de Chile, desde fines del siglo pasado -cuando se enfrenta la tarea de modernizar el ejército- se asume un modelo institucional caracterizado, entre otros aspectos, por la entrega vocacional de sus integrantes, un marcado sentido de cohesión social en torno a la institución y un quehacer orientado por un conjunto de valores, tales como: el honor, el deber, el patriotismo, la lealtad y la disciplina. Dicho modelo se ha mantenido a través del tiempo; más aún, la especialización derivada de los avances tecnológicos ha potenciado la importancia de las funciones, aunque el núcleo básico del sentido de lo militar sigue siendo el conjunto de valores que le da su significado.

Todos estos rasgos han permitido desarrollar un modelo institucional moderno, que no descuida la especialización, pero que mantiene como rasgo esencial su sentido vocacional de servicio a la patria. Refuerza este modelo, en el caso nacional, la conformación mixta de nuestra institución en su dimensión organizacional, en la que se integran profesionales de las armas y soldados conscriptos que cumplen con su servicio militar obligatorio.

\section{MARCO DE LA MODERNIZACIÓN INSTITUCIONAL}

EI sistema de educación militar se encuentra enmarcado en un conjunto de normas legales, disposiciones reglamentarias, órdenes de comando y, en general, documentos de carácter nacional, ministerial e institucional, tanto de tipo directivo como ejecutivo. La concepción fundamental del sistema radica en la educación permanente, puesto que las responsabilidades a las que el profesional de las armas se ve enfrentado así lo requieren.

En el espíritu precedente, y teniendo en cuenta la dinámica interacción que se produce con el cambiante entorno, la institución ha enfrentado un proceso de evaluación y estudio de la estructura, la gestión, los currículos y la tecnología, entre otros aspectos, con el fin de conformar un sistema educacional acorde con el nuevo concepto de ejército.

En cuanto a la relación que deberá existir entre la modernización institucional y la educación militar, se considera fundamental, primero, 
señalar cuáles son las orientaciones expresadas en el "Plan Alcázar", ya que en ellas se encuentra el origen o punto de partida del proceso de cambio.

$\mathrm{Al}$ analizar la educación y la preparación profesional militar², se señala:

- La particular preocupación que constituye la educación superior militar y su futura integración en el nivel respectivo, tanto en el país como en el extranjero, para la obtención de conocimiento actualizado en distintas áreas del quehacer profesional que se pueda utilizar en los procesos educativos propios y, asimismo, aportar al saber de otros.

- El consenso en que el poder ya no proviene sólo de la fuerza y de la posesión de las riquezas materiales sino, fundamentalmente, de la calidad y cantidad de la información que se es capaz de obtener y manejar, la cual está directamente vinculada con el tema del conocimiento y su evolución. Ello obliga a diseñar planes con énfasis en el autoaprendizaje, la capacitación, y el estímulo de la capacidad creadora.

- El sistema docente del ejército es fundamental si se tiene presente que la educación es una de las áreas donde se inicia todo proceso que aspire a proyectar cualquier organización.

- Los oficiales y clases deben propender cada vez más a la especialización, lo que, unido a una mayor permanencia en la institución, permitiría un progreso sostenido. Por lo tanto, los convenios con entidades técnicas y de educación superior son muy importantes.

\section{MODERNIZACIÓN Y EDUCACIÓN MILITAR}

Diferentes y muy variadas son las definiciones que, desde diversos campos del saber, se dan al concepto de modernización. Sin embargo, lo apropiado es aproximarse al tema de manera que su relación con la educación quede de manifiesto.

2 Comando de Institutos Militares, Marco Teórico del Proyecto de Actualización del Proceso de Educación, Instrucción y Formación, Santiago, Chile, 1994. 
Las sociedades modernas se caracterizan por la aparición de nuevos conocimientos; esto presupone la existencia de hombres con una creciente capacidad para entenderlos y aplicarlos a los procesos productivos que desarrollan. Se comprende, entonces, que la condición básica de la modernización es la capacidad del individuo para adaptarse a las cambiantes circunstancias del contexto social. Este proceso de adaptación requiere de instituciones sociales y políticas, de procesos y formas de comportamiento que permitan una constante introducción de nuevos productos, nuevas carreras y nuevos estándares de conducta social.

¿Cómo debe responder la estructura educativa a este desafío?

Debe proporcionar al individuo una adecuada apertura y disposición para buscar opciones, aceptar nuevas ideas y explorarlas. Básicamente, estimular una disposición mental permanente, continua y creciente. Asimismo, debe despertar su interés en participar con una visión futurista. En general, la respuesta se divide en dos componentes: por una parte, modernización de la estructura, orientación y fines de los procesos educativos; por otra, renovación (y en algunos casos innovación) de contenidos, métodos, materiales y currículo, entre otros aspectos.

En relación a la estructura, se trata de reconocer la interdependencia orgánica de los diferentes niveles de enseñanza que, en una especie de continuo lineal, coadyuvan a la formación del profesional del Ejército. Se requiere, primero, modernizar la conceptualización y las bases teóricas de cada subsistema y nivel educacional en particular, considerando lo peculiar de sus propósitos, para asegurarse de que la corriente innovadora tenga raíces profundas y sea perdurable en el tiempo.

El segundo aspecto dice relación con la calidad del servicio educativo que se ofrece, siendo, a todas luces, más determinante como factor de modernización. Éste exige que el sistema educativo esté en manos de personal idóneo -de por sí innovador y creativo-, que cumpla los diversos roles que la ejecución de las políticas y la gestión, tanto educacional como administrativa, implican. La innovación debe producirse en procedimientos, metodologías, concepciones, actitudes y nuevas especializaciones. 
Si la modernización de políticas educativas es un hecho constante, si los fines del sistema educativo se actualizan permanentemente, si la estructura que sustenta a dicho sistema se hace más funcional y operativa, si el personal que desempeña diversos roles de esa estructura se moderniza -especialmente a nivel de actitudes-, el problema se resuelve parcialmente. La otra parte debe abordarse como proceso específico, directamente relacionado con la formación de actitudes y valores, con la transmisión de información, habilidades y destrezas. La reforma en este sector debe ser de tal magnitud que la innovación permanente y propagadora constituya un sello institucional en cualquiera de los niveles y ocupaciones de la profesión militar.

Por otro lado, no hay que olvidar que la calidad del conocimiento que aporta un sistema educativo está determinada por la calidad de la información que se provee al estudiante (a través de instructores, profesores, medios de apoyo pedagógico y experiencias de aprendizaje) y por el contexto institucional de cada uno de sus componentes. Esta calidad debe caracterizarse por ser completa, actualizada, precisa, apropiada, gradual y relevante; debe también estar de acuerdo con los objetivos que persigue el currículo y con el perfil del egresado, pues ella puede reforzar un valor o una actitud o puede ser un instrumento para un conocimiento posterior.

En resumen, el contexto institucional debe modernizarse en: qué enseñar, cómo enseñar, quién enseña, quién dirige o administra, quién supervisa, quién asesora, cuándo y dónde enseñar y para qué enseñar.

\section{La calidad educativa}

Se requiere abogar por concepciones globales y flexibles que permitan un adecuado movimiento dentro de condiciones nuevas. Se necesita un empuje especial en la educación para el logro de un mejor desarrollo de la mente del individuo. En la actualidad ha quedado de manifiesto que se requiere cada vez más capacidad para poder actuar, decidir y orientarse ante situaciones inesperadas, confusas y de gran incertidumbre, que exigirán la aplicación inteligente de los conocimientos adquiridos. Mientras el bagaje de conocimientos no puede seguir siendo una medida cuantitativa (cada vez más y más 
información), sino que debe enfocarse a la selección adecuada, la necesidad de desarrollo de la capacidad mental se hace más evidente.

La educación no puede estar apoyada por un volumen informativo en ascenso incontenible, que se traduce en mayor número de temas y de asignaturas. ¿Qué educación y qué proceso de enseñanza serán entonces los que conduzcan a las expectativas de mejoramiento? Aquellos que conjuguen la formación de destrezas mentales para asimilar y aplicar conocimientos y la constitución de un individuo con personalidad amplia, curioso y estimulado por las realizaciones, que base sus elecciones y decisiones en argumentos sólidos, con gran imaginación y capacidad de actuar en forma aislada o en grupo ${ }^{3}$.

Tres serían, en consecuencia, los aspectos fundamentales a las que se orientaría un óptimo proceso educativo: el saber, la reflexión y la acción. Además, debe haber un claro énfasis en el proceso de enseñanza y no en el producto, es decir, en lo formativo por sobre lo informativo.

Características de la organización educativa

La preocupación fundamental de esta organización debe centrarse en lo propiamente educativo, ubicando el ámbito administrativo sólo como apoyo a la gestión educacional. Esto, que es aparentemente obvio, ha sido muchas veces postergado en beneficio de aspectos disciplinarios y de control, y de otros objetivos que en determinados momentos aparecen como superiores.

Es vital para el adecuado funcionamiento, permanencia y crecimiento de ésta, su constante intercambio de energía, información y recursos con el medio institucional en que está inserta. La organización educativa debería ser la principal gestora, facilitadora e impulsora de las actividades que tengan estos propósitos.

En cuanto a su funcionamiento, la organización educativa adquiere características propias de un enfoque sistémico abierto, es

3 Comando de Institutos Militares, Proyecto Global de Reestructuración del Sistema Docente Institucional, Fase Idea, Santiago, Chile, 1996, p.10 
decir, se encuentra en relación dinámica y compleja con su medio ambiente del que recibe constante energía y que modifica su accionar en virtud del logro y optimización de los objetivos que orientan su acción. Contrariamente a lo que sucede en un sistema cerrado, el sistema abierto no es autosuficiente ni independiente. La relación dinámica con el entorno permite recibir energía de entrada -que el sistema transforma a través de diferentes procesos-, que luego se entrega al mismo medio en forma de productos.

\section{Organización y desarrollo curricular}

Corresponde a la organización educativa diseñar y desarrollar todo aquello que ofrece al alumno como posibilidad de aprender. En la actualidad, la oferta y las oportunidades educativas se comprenden en la noción de currículo, concepto que reúne principios, procedimientos y actitudes, y que abarca, además, tanto los medios a través de los cuales la organización proporciona esas oportunidades como aquellos por los que evalúa los procesos mismos de enseñanza aprendizaje. En el currículo se plasman las intenciones educativas.

Ahora bien, ¿a qué debe responder su formulación?

- A las necesidades de la institución en cuanto a conocimiento y capacitación profesional, sin olvidar que, como ningún otro sistema social, la acción educativa se encuentra inserta en un amplio sistema valórico, de carácter particular.

- Al convencimiento de que la educación se encuentra determinada por las características sociales en que se desenvuelve la vida humana. Esto implica que los actores del proceso educativo reciben variadas influencias que condicionan sus expectativas y que, en definitiva, se convierten en imperativo para el sistema. El currículo debe partir de intereses detectados, a la vez que desarrollar nuevos.

- Al dinámico crecimiento y profundidad de las necesidades educativas, que obliga al sistema a plantear constantemente soluciones eficientes a las diferencias entre lo que realmente se está entregando y lo que se debiera entregar. 
- Al desafío de capacitar al hombre no sólo para su accionar en relación con la sociedad civil. En este sentido, entonces, se plantea la necesidad de estimular y orientar la formación de hombres capaces de adaptarse y contribuir al carácter multifacético y técnico de la sociedad actual.

- A la necesidad de sustentar al sistema sobre una concepción operativa que, junto con responder a los requerimientos contemporáneos (carácter flexible), otorgue una base de conocimiento firme para que el educando pueda sentirse seguro.

\section{EDUCACIÓN MILITAR}

El objetivo de la educación militar es entregar al ejército -y a la sociedad-oficiales, suboficiales y clases con sólidos principios y valores militares, capaces de actuar como líderes en unidades altamente entrenadas, para enfrentar un mundo cada vez más tecnificado, dinámico y complejo.

Aunque estemos conscientes de que este objetivo constituye una actualización de lo que cualquier ejército aspira a lo largo de su existencia, lo central del mismo es equilibrar cambio y continuidad, de forma de mantener la esencia de un ejército institucional, de perfil vocacional y ajustado a la realidad nacional, pero avanzado en cuanto a conocimientos, procedimientos, gestión y sistemas de armas.

Para lograr este objetivo se definieron los siguientes parámetros:

- Clara definición de roles directivos y ejecutivos, permitiendo determinar misiones, áreas de acción y responsabilidades.

- Descripción de líneas de carrera, permitiendo a cada integrante de la institución diseñar sus proyectos profesionales, de acuerdo con las necesidades del ejército.

- Incremento programado de oportunidades de especialización.

Los cambios generados en educación han permitido ordenar y señalar funciones específicas a diversos organismos de la institución. Producto de esto, nació la Dirección de Educación del Ejército, organismo encargado de: "Asesorar al mando en la dirección del sistema educacional militar. Su función principal es entregar las políticas 
educacionales e integrar, de manera planificada y coordinada, los subsistemas de docencia militar, instrucción militar, entrenamiento y capacitación para el cumplimiento de sus funciones específicas y la misión institucional".

La necesidad de pasar de una planificación centralizada a una coordinada, a través de sus subsistemas, requirió la formulación de dos estrategias:

1. La descentralización curricular, supone que el organismo central entrega la responsabilidad a los institutos militares para proyectar y planificar sus procesos educativos, ejecutándolos de acuerdo a las políticas generales.

- Formular normas y establecer sistemas que aseguren eficiencia, eficacia y, a la vez, permitan innovar y operar en forma flexible en materias de currículos, programas y proyectos de investigación y extensión.

- Establecer sistemas eficientes de gestión que hagan posible asignar presupuestos globales a las distintas escuelas y academias y permitan reasignar recursos de acuerdo con el plan de desarrollo estratégico y sus proyectos.

- Implementar los mecanismos de control de gestión que aseguren, en un esquema descentralizado, la eficiencia de las instituciones formadoras y la coherencia entre sus actividades y las estrategias globales del ejército.

2. La desconcentración administrativa, como un proceso en que se delegan las funciones desde el organismo central de educación hacia los institutos militares, ejecutando éstos las misiones de acuerdo con el nivel de responsabilidad y los medios presupuestados ${ }^{4}$.

- Racionalizar, tecnificar y profesionalizar la administración docente para que preste eficiente servicio y proporcione un apoyo eficaz, al desarrollo curricular de las escuelas y academias.

- Implementar un nuevo sistema de gestión de recursos humanos que contemple una política de reconocimiento y compensación

4 Comando de Institutos Militares, Sistema Educativo Institucional: Gestión Docente, Santiago, Chile, 2000, p. 104. 
al buen desempeño profesional, que motive y estimule el apoyo eficiente a la actividad docente, estableciendo mecanismos y objetivos transparentes de evaluación, incentivos o sanciones según desempeño, oportunidades de capacitación y perfeccionamiento permanentes.

La estructura del sistema educacional está determinada por la carrera militar, en una preparación progresiva y sistemática determinada por los siguientes niveles:

- La etapa formativa, representada por las Escuelas Matrices. Enseña los conocimientos generales de carácter humanista, científico y tecnológico, y los básicos instrumentales de las ciencias militares, junto con sólidos valores y actitudes, sustento del particular sistema del Ejército. Al mismo tiempo, la Escuela Militar posibilita el inicio de una carrera de pregrado y su continuidad según la capacidad individual.

- La segunda etapa tiene lugar en la educación de las Escuelas de Armas, de los Servicios y Especialidades Secundarias, cuyo nivel de perfeccionamiento profesional y de especialización técnica marca la diversidad de funciones que adquiere la organización y que exige, en algunos casos, una capacitación de breve duración, centrada en una preparación de tipo práctico o una especialización de alto nivel.

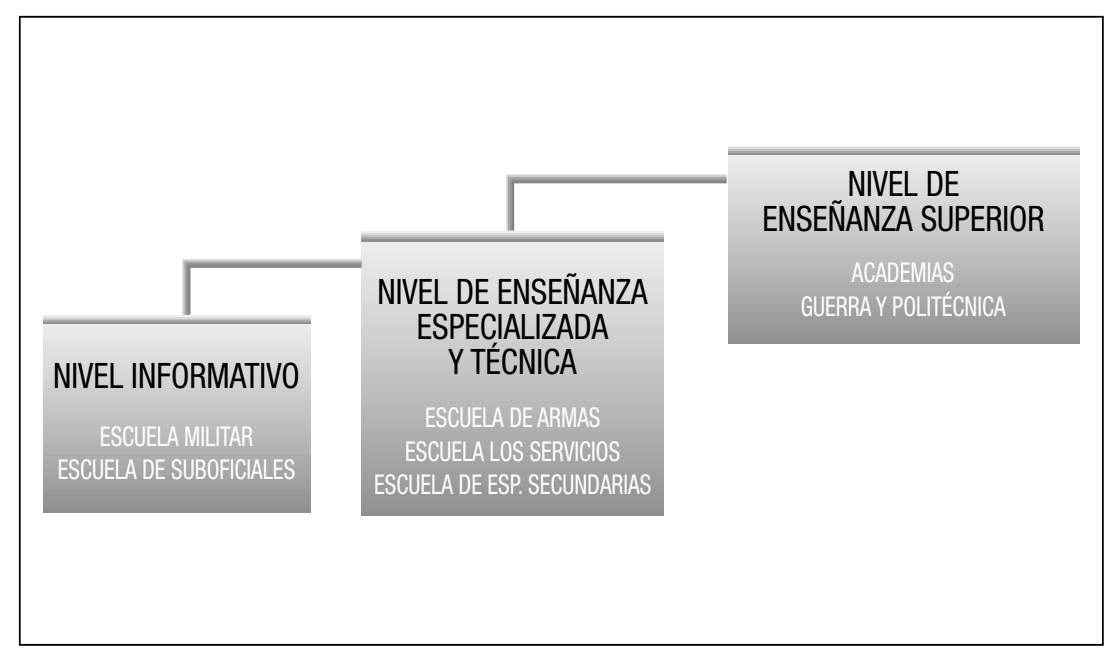


- La tercera etapa, representada por las Academias de Guerra y Politécnica Militar, tiene la responsabilidad de la formación de posgrado de la educación superior.

El sistema educacional del ejército contempla, como elemento básico, una formación militar, sustentada en la normativa legal establecida para todas las FF.AA. y, en particular, para su modelo educacional vigente. La estructura de este modelo se basa en los principios de coherencia y gradualidad (jerarquía), de modo análogo a la enseñanza civil como se grafica a continuación.

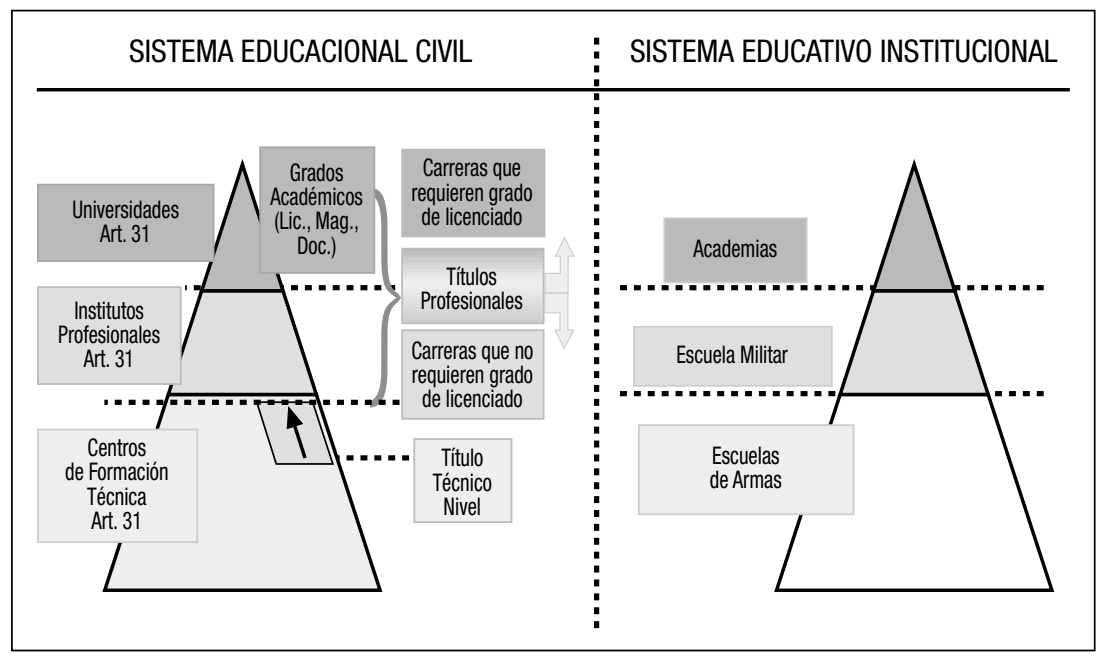

La concepción educacional parte de un enfoque centrado en la persona, que propone que el educando debe participar de su propio proceso, como sujeto consciente, para ir desarrollando las capacidades propias con sus dimensiones individuales, tanto de carácter afectivovalórico como de conocimiento y psicomotriz.

Este enfoque propone:

- Alcanzar la formación de un militar que reúna los fundamentos de la profesión, vale decir, equilibrio e integración entre los valores militares, capacidad física, conocimiento de la ciencia 
militar y, por último, formación en ciencia y tecnología. Con ello se pretende evitar procesos educativos compartimentados.

- Aplicar un nuevo concepto de educación militar integrando a la preparación profesional, instrucción, entrenamiento y capacitación a través de la docencia. De esta forma se erradica el antiguo concepto que limitaba la enseñanza a las actividades en aula.

- Definir un sistema formativo y de preparación que obedezca a los principios de secuencialidad, progresión y permanencia, siendo este último uno de los requisitos fundamentales para el éxito del sistema mismo. Así se evitan pérdidas de esfuerzos, enfatizándose un uso intensivo y programado de los periodos interdocentes.

- Conformar un proceso de gestión descentralizada de los aspectos curriculares y administrativos, permitiendo asignar a la etapa y organismo correspondientes los mecanismos de responsabilidad, evaluación y administración. Esto permite descongestionar, racionalizar, tecnificar y profundizar la gestión educativa, permitiendo un eficiente empleo de sus recursos humanos y financieros.

- Desarrollar en cada instituto un proyecto educativo, esto es, un documento integral que contenga los lineamientos generales y particulares, a través de los cuales desarrollan su labor educativa. 


\section{REFERENCIAS BiBLIOGRÁFICAS}

Cheyre Espinosa, Juan Emilio, "Tendencias de la Educación Militar en el marco del cambio en el conocimiento", en Memorial del Ejército de Chile N446, CESIM, Santiago, Chile, 1995.

Comandancia en Jefe del Ejército, Directiva Particular de Instrucción, Entrenamiento y Docencia, Santiago, Chile, 1996.

Comando de Institutos Militares, Marco Teórico del Proyecto de Actualización del Proceso de Educación, Instrucción y Formación, Santiago, Chile, 1994.

Comando de Institutos Militares, Proyecto Global de Reestructuración del Sistema Docente Institucional, Fase Idea, Santiago, Chile, 1996.

Comando de Institutos Militares, Sistema Educativo Institucional: Filosofía, Políticas y Alcances, Santiago, Chile, 1997.

Comando de Institutos Militares, Sistema Educativo Institucional: Gestión Docente, Santiago, Chile, 2000. 
